\title{
The Qujing incoherent scatter radar: system description and preliminary measurements
}

\author{
ZongHua Ding ${ }^{*}$, Jian Wu, ZhengWen Xu, Bin Xu and LianDong Dai
}

\begin{abstract}
The Qujing incoherent scatter radar (QJISR), the first one in China with the geographic location $\left(25.6^{\circ} \mathrm{N}, 103.8^{\circ} \mathrm{E}\right)$, was brought into operation since the spring of 2014. The QJISR was a mono-static pulsed radar working in the operating frequency $500 \mathrm{MHz}$, the peak power 2 Megawatt, and a 29-m steerable parabolic dish. This paper mainly presents the basic configuration and implementation of QJISR, including the antenna, transmitter, receiver, signal processing, and data analysis. Some preliminary observation results are also reported including the raw echo, power spectra, and its ionospheric parameters: electron density, electron temperature, ion temperature, and drift velocity.
\end{abstract}

Keywords: Incoherent scatter radar, Ionosphere, Echo profile, Power spectra, Electron density, Electron temperature, Ion temperature

\section{Introduction}

The incoherent scatter radar (ISR) owns some unique advantages including the wider coverage and simultaneous measurements of multi-parameters, which provides an outstanding opportunity to investigate the ionospheric phenomena and its physical process. Gordon (1958) proposed to use the Thomson scatter of free electron to sound the ionosphere on the ground by the powerful radar. In the same year, Bowles (1958) successfully carried out the first incoherent scatter experiment using a radar with peak power $6 \mathrm{MW}$ and central frequency $41 \mathrm{MHz}$. Bowles' results found approximately the expected amount of power scattered from the electrons but one much narrower the spectral width, which implies that the scatter is not fully independent since the heavier and slower ions are controlling the scattering spectral. Afterward, the ionospheric incoherent scatter technique is widely used in the world (Evans 1970).

As the earliest ISR, the Arecibo (Ioannidis and Farley 1972) and Jicamarca (Farley 1991) radars started the routinely operation since 1962 . Nowadays, there are more

*Correspondence: zhdingmou@163.com

China Research Institute of Radio-wave Propagation, 36 Xianshan Road, Chengyang District, Qingdao 266107, China than ten ISRs in the world, which are mainly constructed in America and the Northern Europe (European Incoherent Scatter Scientific Association, EISCAT) separately (Evans 1969; Huuskonen et al. 1987; Kelly et al. 1995; Wannberg et al. 1997; Ya and Zhivolup 2013). In Asia, the middle and upper atmosphere (MU) radar was completed at Shigaraki $\left(34.8^{\circ} \mathrm{N}, 136.1^{\circ} \mathrm{E}\right)$ in 1984 (Susumu et al. 1984) and had played an important role in the research of middle and upper atmospheric dynamics in Japan. At present there is no ISR available in the low latitude of Asia, in which the ionosphere has some unique characteristic compared with the American sector such as the known east-west longitudinal differences (Zhao et al. 2013). The southwest of China is located in the northern crest of the equatorial ionization anomaly (EIA) and near to the Qinghai-Tibet plateau, in which some important science cases have not completely resolved including the evolution of EIA and the coupling between the atmosphere and ionosphere.

Supported by the Space Weather Monitoring Meridian Project of China (Wang 2010), China Research Institute of Radio-wave Propagation (CRIRP) started the construction of Qujing incoherent scatter radar (QJISR), the first one in the mainland of China, in January 2008. After more than 6 years of effort, CRIRP completed the 
construction and started the routine operation from March 2014 (Ding et al. 2014a). The QJISR is located in Zhanyi county $\left(25.6^{\circ} \mathrm{N}, 103.8^{\circ} \mathrm{E}\right.$, with the altitude of $2040 \mathrm{~m}$ ), Yunnan Province, China. The geomagnetic latitude is $14.3^{\circ} \mathrm{N}$, which is just near to the northern crest of EIA. The QJISR is one of the key instruments in the Meridian Project which consists of diverse ground-based remote sensing facilities aligned near the longitude $120^{\circ} \mathrm{E}$ for space environment monitoring and forecasting.

This paper presents a detailed technical description of the QJISR system and its preliminary observations for the first time.

\section{QJISR technical description}

The QJISR is a $500-\mathrm{MHz}$ mono-static pulse-modulated radar with a $29-\mathrm{m}$ fully steerable parabolic dish and a 44-m radome. The technical parameters are listed in Table 1. The overall block diagram is presented in Fig. 1. Except that the transmitter is imported from USA, all the other subsystems are designed and manufactured in China.

The operation process of the QJISR can be summarized as follows. The phase-modulated pulse is generated by a direct digital synthesis (DDS) exciter unit and then is mixed with the reference oscillation frequency $(30 \mathrm{MHz})$ to be up-converted to radio frequency $500 \mathrm{MHz}$ with the power level of $10 \mathrm{dBm}$, which will act as the signal to excite the subsequent power amplifier. The exciter signal is first amplified by a solid-state amplifier to about $100 \mathrm{w}$ and then amplified by the pulsed klystron to the high power of $2 \mathrm{MW}$. The RF output power from the transmitter goes through the wave guide, azimuth and elevation knot, T/R switch, and orthogonal polarizer, and finally reaches the feeder, where the RF signal is radiated into the ionosphere. The radio signal will be scattered by

Table 1 Technical specifications of the QJISR

\begin{tabular}{ll}
\hline Item & Specifications \\
\hline Frequency & $500 \mathrm{MHz}$ \\
Peak power & $2 \mathrm{MW}$ \\
RF duty cycle & $\leq 5 \%$ \\
System temperature & $150 \mathrm{~K}$ \\
Pulse width & $20-500 \mu \mathrm{s}$ \\
Pulse repetition period & $1-20 \mathrm{~ms}$ \\
Modulation type & $13-$ bit Barker, 16-bit Alternative code \\
Antenna & Parabolic dish with the 29 m aperture \\
Antenna gain & $41 \mathrm{~dB}$ \\
Side lobe & $17.5 \mathrm{~dB}$ (first) \\
Beam width & $1.3^{\circ}$ \\
Slew rate & $3 \% / \mathrm{s}$ (elevation),5\%s (azimuth) \\
\hline
\end{tabular}

the ionosphere, parts of which are detected by the radar receiving antenna. The received RF signals are bandpass-filtered and low-noise-amplified in the analogue front end and then transferred to the back-end receiver located in the control room. Here the RF signal is amplified and down-converted to the intermediate-frequency (IF) $30 \mathrm{MHz}$ with the bandwidth $5 \mathrm{MHz}$. The IF signals will be digitized, processed, and stored by the signal processor. And the echo profile and power spectrum of the ionosphere are output to the data terminal computer, from which the ionospheric parameters including the electron density, electron and ion temperature, plasma drift velocity can be derived. Binary phase codes including the 13-bit Barker code and 16-bit alternative code are utilized for pulse compression by which the signal-tonoise ratio can be improved with better range resolution. The sequence of 13-bit Barker code is 1,1,1,1,1,-1,-1,1, $1,-1,1,-1,1$. Here ' 1 ' and ' -1 ' represent the phase 0 and 180 , respectively. And the 16-bit alternative code is the same to that used by EISCAT (Lehtinen and Haggstrom 1987; Lehtinen and Huuskonen 1996; Sulzer 1993; Markkanenet al. 2008).

Since there is a lightning rod and a small steel plate used for the elevator at the top of the radome, the maximal elevation cannot be set to $90^{\circ}$ so as to avoid the strong reflections. The minimal elevation is set to $10^{\circ}$ to avoid the influence of clutter from the ground and mountain reflection.

\section{Antenna}

Figure 2 shows the radome with the diameter of $44 \mathrm{~m}$ and the parabolic dish with the aperture of $29 \mathrm{~m}$. The radome is made of organic reinforced plastics and consists of about 1600 honeycomb-like sub-plates with the thickness $0.2 \mathrm{~m}$. The radio attenuation of the radome is estimated be as $0.3 \mathrm{~dB}$ in $500 \mathrm{MHz}$. The antenna is a Cassegraintype parabolic dish of which the total gain is about $41 \mathrm{~dB}$. Figure 3 shows the measured antenna diagram, from which the first side lobe $-17.58 \mathrm{~dB}$ and the $3 \mathrm{~dB}$ beam width can be known. A calibration tower about $3 \mathrm{~km}$ away is used to perform the antenna pattern test and calibration.

\section{Transmitter}

Figure 4 shows the overview of the transmitter, which is designed and manufactured by the Diversified Technologies Inc, USA. It consists of the pre-amplifier, the cathode-modulated pulsed klystron, solid-state modulator, capacitor bank, high voltage-power supply, voltage regulator and distribution box, water-cooling manifolds, three-port circulator, harmonic filter, monitoring and controlling equipment. For safety, all the components with high voltage are stored inside the oil tank. The 

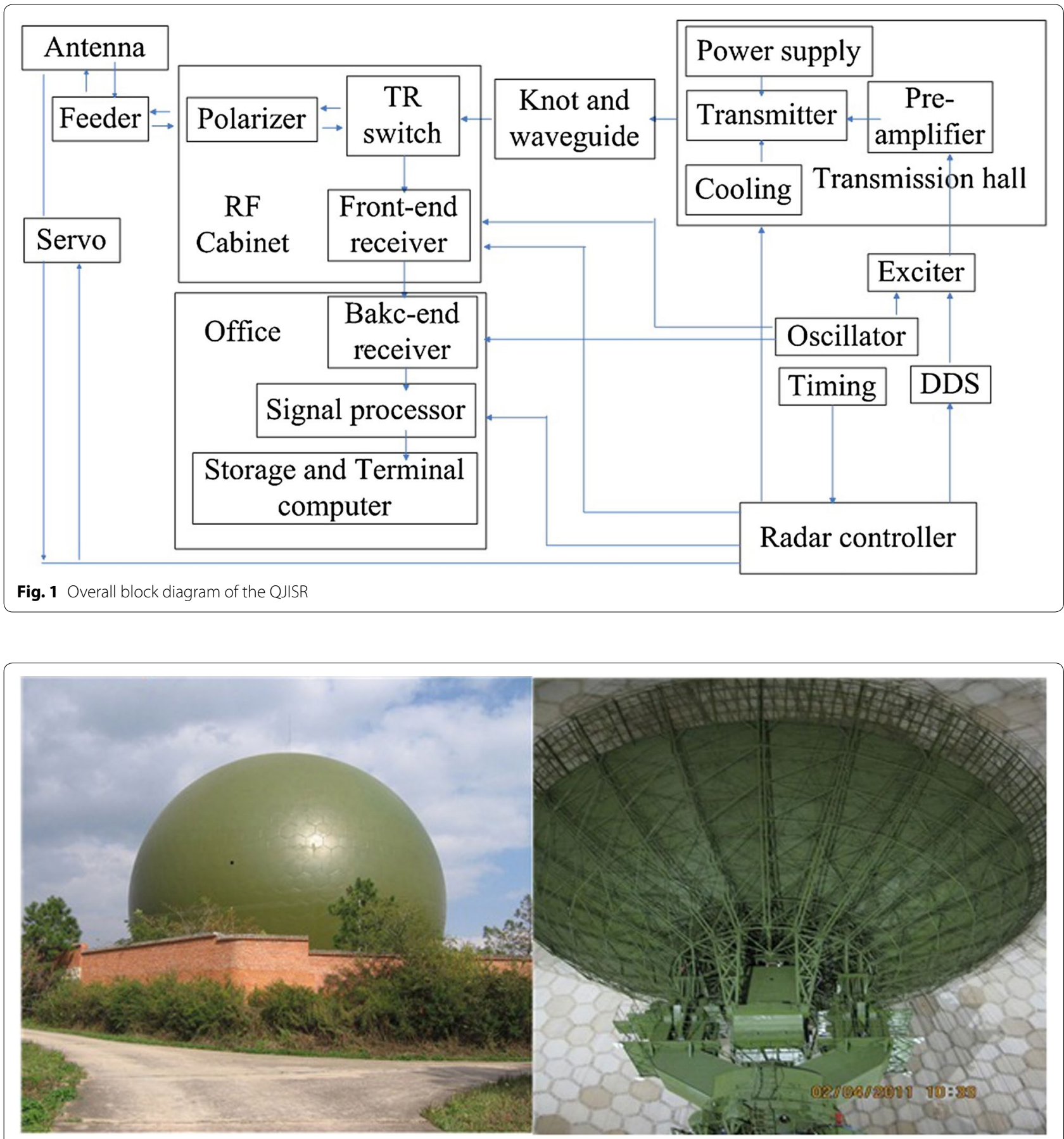

Fig. 2 Radome (left) and the parabolic dish (right)

water-cooling manifolds perform the real-time monitoring of the water temperature, pressure and flow. Once any faults occur, the high-voltage system is shut down and the alarm information is shown immediately in the computer touch screen.

Figure 5 is the principal diagram of the transmitter. The blank circle represents the three-port circulator, which is used to protect the klystron from the reflected power signal. The directional coupler acts as a waveform and power monitoring of the output RF signal. The harmonic filter is used to filter out the harmonic signal from the klystron output such as the components at 1000 and $1500 \mathrm{MHz}$. The maximal output power and RF duty cycle are $2 \mathrm{MW}$ and $5 \%$, respectively. The cathode voltage and current of 


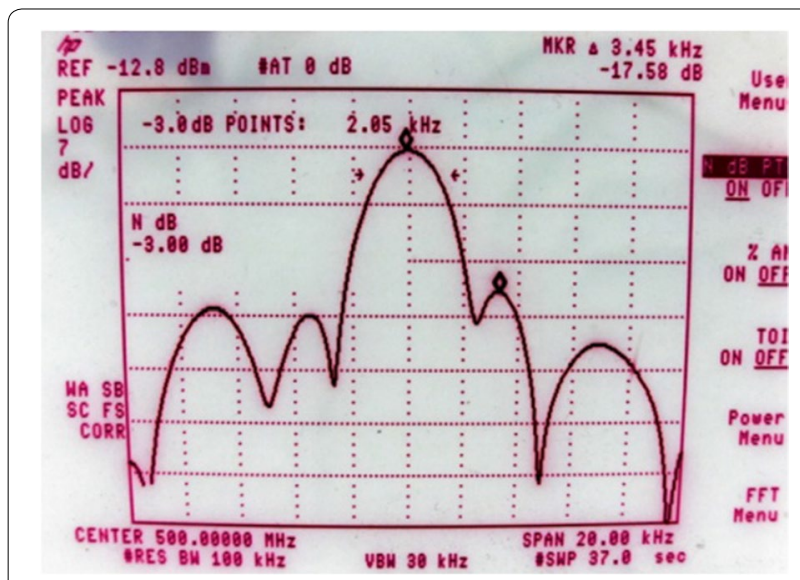

Fig. 3 Measured antenna pattern

the klystron are about $100 \mathrm{kV}$ and $45 \mathrm{~A}$ respectively. The driving signal $(10 \mathrm{dBm})$ coming from the exciter is first amplified to about $100 \mathrm{~W}$ and then input to the klystron.

Figure 6 is the monitoring software from which we can make the settings, run the transmitter, and monitor its status. The monitoring software is also developed and integrated inside the transmitter by the Diversified Technologies Inc. All the real-time parameters including the peak power ( $93 \mathrm{dBm}$, which is equal to $2 \mathrm{MW}$ ), high

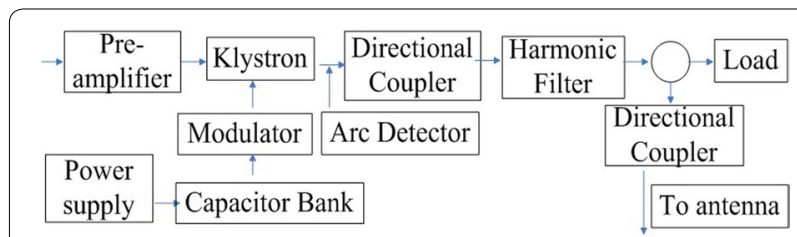

Fig. 5 Schematic diagram of the transmitter RF chain

voltage $(-98 \mathrm{kV})$ and duty cycle $(3.5 \%)$ can be known from the software interface.

The modulator is built from a series stack of integrated insulated gate bipolar transistors (IGBT), which can provide the $100 \mathrm{kV}$ pulsing capability as well as the fast fault protection. These switches, fully isolated from ground, can be quickly and arbitrarily closed or opened providing sub-microsecond removal of stored energy. Using the above architecture, the high-voltage transformer and crowbar are unnecessary, which greatly reduce the available arc energy and provide the greater reliability.

\section{Receiver}

The scattered echoes from the ionosphere are very weak, which are usually much lower than the noise level. So a high-gain and low-noise receiver is needed, which

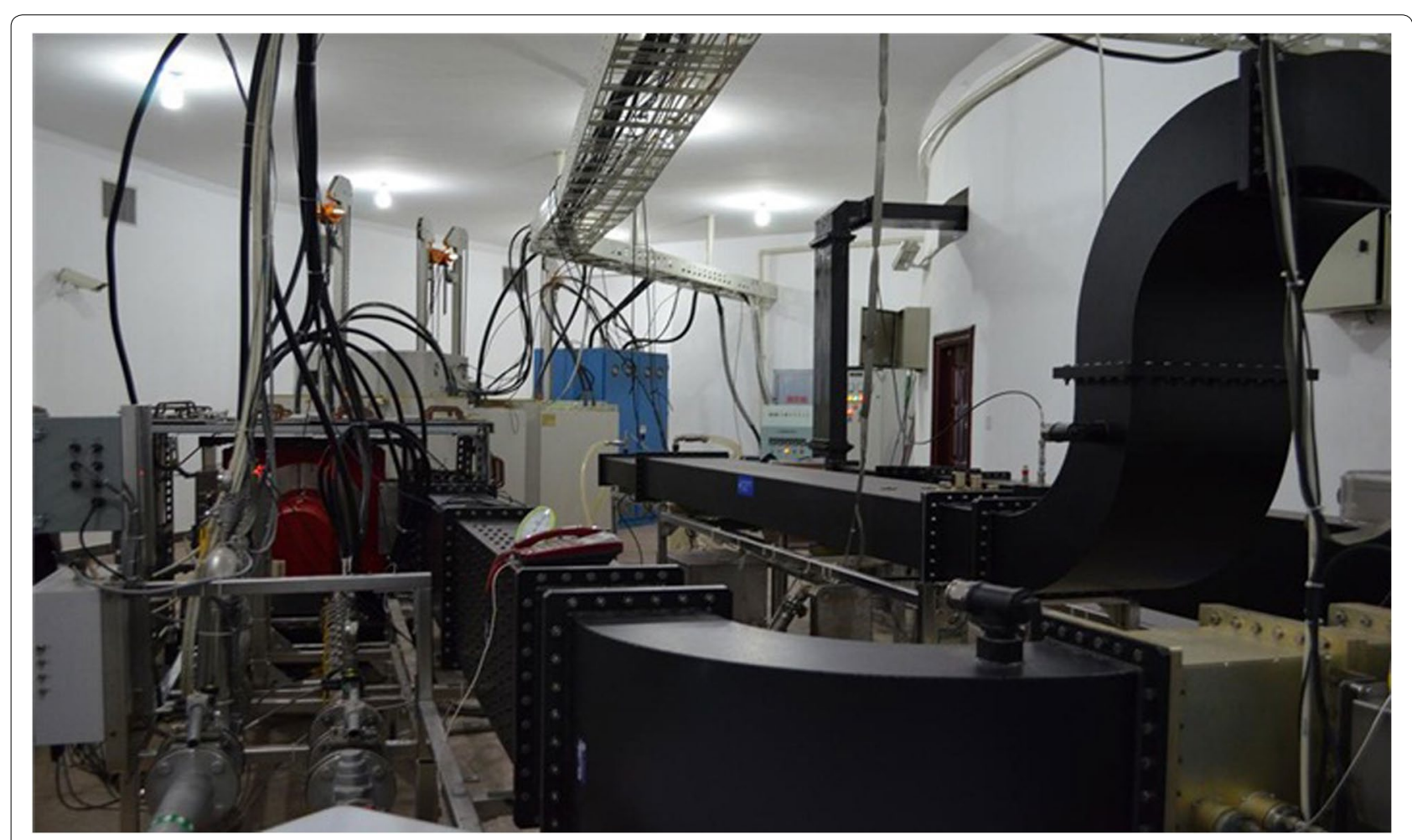

Fig. 4 Photograph showing the overall view of the transmitter 


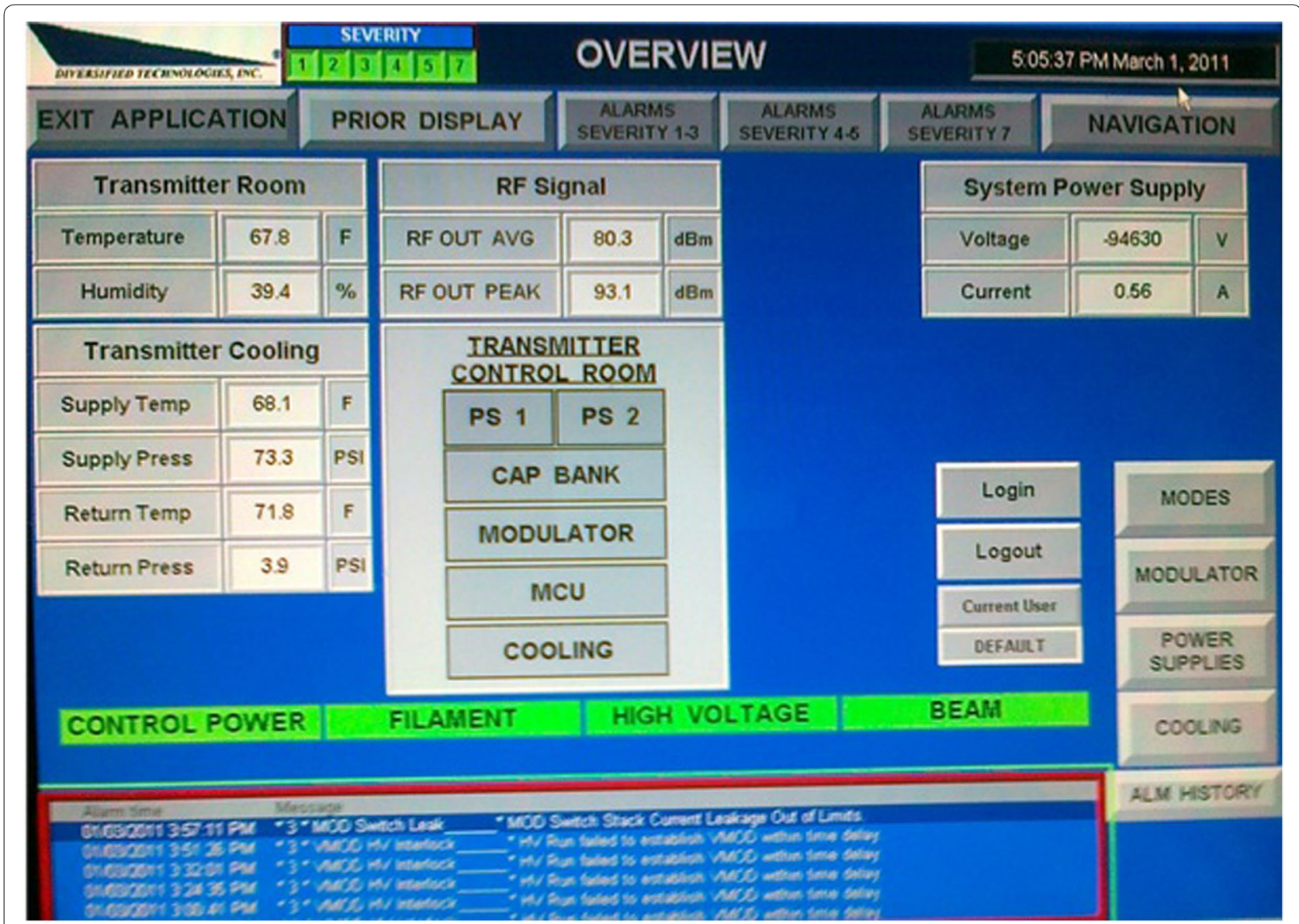

Fig. 6 Monitoring software interface of transmitter

\section{$\rightarrow$ IF Signal $\rightarrow$ ADC $\rightarrow$ DDC $\rightarrow$ Filter $\rightarrow \begin{aligned} & \text { Lag Profile } \\ & \text { Matrix }\end{aligned}$ \\ Data output $\longleftarrow$ Integration $\longleftarrow$ FFT ACF Calculation}

Fig. 7 Schematic diagram of the signal processing system

includes two parts: the front end near to the feeder and the back end located in the controlling room. The total gain of the receiver is more than $90 \mathrm{~dB}$ which can be adjusted by the variable attenuator. The dynamic range of the receiver is more than $70 \mathrm{~dB}$, since the ionospheric scattered echo in the different conditions are highly variable.

The front-end receiver consists of the polarizer, TR switch, low-noise amplifier (LNA), and filters. The polarizer will switch the left- and right-hand circular wave with the isolation of above $30 \mathrm{~dB}$. The TR switch is used to protect the receiver from the high-power leakage from the transmitter route with the isolation more than $60 \mathrm{~dB}$, which guarantee its output signal much less than the maximal input level of the LNA. The LNA will amplify the weak echo with the gain $40 \mathrm{~dB}$. There is a test port inside the front-end chain, which is used to inject the test signal to measure the receiver performance characteristic and help fault diagnosis.

The back-end receiver consists of band-pass filter, down-converter, and low-noise amplifier. The $500-\mathrm{MHz}$ $\mathrm{RF}$ echo signal from the front-end receiver is down-converted to the first IF $140 \mathrm{MHz}$ (the first local oscillation frequency $360 \mathrm{MHz}$ ) and then the second IF $30 \mathrm{MHz}$ (the second oscillation frequency $110 \mathrm{MHz}$ ) with the level $10 \mathrm{dBm}$ and bandwidth $5 \mathrm{MHz}$, then output to the analog-to-digital converter (ADC) in the signal processor.

\section{Signal processor}

The signal processor of QJISR consists of the ADC, digital signal processor (DSP), buffer memory, clock, and power supply. The algorithm flowchart include the 
post-detection filter, lag profile matrix calculation, autocorrelation function calculation, fast Fourier transform (FFT), and incoherent integration as shown in Fig. 7.

The $30-\mathrm{MHz}$ IF analog signal from the back-end receiver is sampled at $40 \mathrm{MHz}$ with 16 bit of resolution and then digital-down-converted to base band. One box filter is used to remove the noise outside the spectra band and reduce the data rate of the complex sampling for the next real-time computations. The calculation of autocorrelation function (ACF) is complicated and the details can be referred from (Nygren 1996). So the lag profile matrix is adopted for the convenience of the ACF calculation.

Formula (1) is the ACF expression of the long pulse, in which the ' $i$ ' denotes the order of the sampling point, i.e., the height or range. The $x$ represents the complex sampling data and $v$ is the coefficient responsible for the number of lag product in zero lag. Figure 8 shows the schematic diagram of the lag profile matrix. The black dots aligned along the diagonal line are used to calculate the $\operatorname{ACF}(i, k)$.

$\operatorname{ACF}(i, k)=\frac{1}{k+v} \sum_{j=i}^{i+k+v-1} x_{j-k} \cdot x_{j}^{*}, \quad k=0,1, \ldots, n-1$
We assume that the pulse length is $6 \mathrm{~T}$, the sampling interval is $T$ and $v$ is equal to 3 . The product $x_{i-j} \cdot x_{j}^{*}$ is called the lagged product. The different lagged product covers the different range span (represented by $i$ ). The range span of zero lag is 6 . The range span of 1st lag is 5 and so on. For zero lag, there are three lagged products. And for first, second, and third lag, there are four, five, and six lagged products, respectively. Thus the measured $\mathrm{ACF}$ in the different lags coves the same range span, which helps improve the calculation stability.

The measured $\mathrm{ACF}_{\mathrm{m}}(i, k)$ represents the ACF estimates in the $i$ th range $(r)$ and $k$ th lag $\left(t^{\prime}-t^{\prime}\right)$, which is related to the ionospheric scatter $\mathrm{ACF}_{\text {ion }}$ using the formula (2). Then the ionospheric incoherent scatter spectra can be estimated from the $\mathrm{ACF}_{\text {ion }}$.

$$
\mathrm{ACF}_{m}=k \cdot \mathrm{ACF}_{\text {ion }}\left(t-t^{\prime}, r\right) \cdot \int_{-\infty}^{\infty} W_{t, t^{\prime}}^{r}(S) \cdot \mathrm{ds}
$$

The $W_{t, t^{\prime}}^{r}$ in (2) is the range ambiguous function, which is determined by the transmission pulse waveform and receiver response function $h(t)$ as the formula (3).

$$
W_{t, t^{\prime}}^{r}(S)=[h(t) \otimes e(t-S)] \cdot\left[\overline{h\left(t^{\prime}\right) \otimes e\left(t^{\prime}-S\right)}\right]
$$

(1)

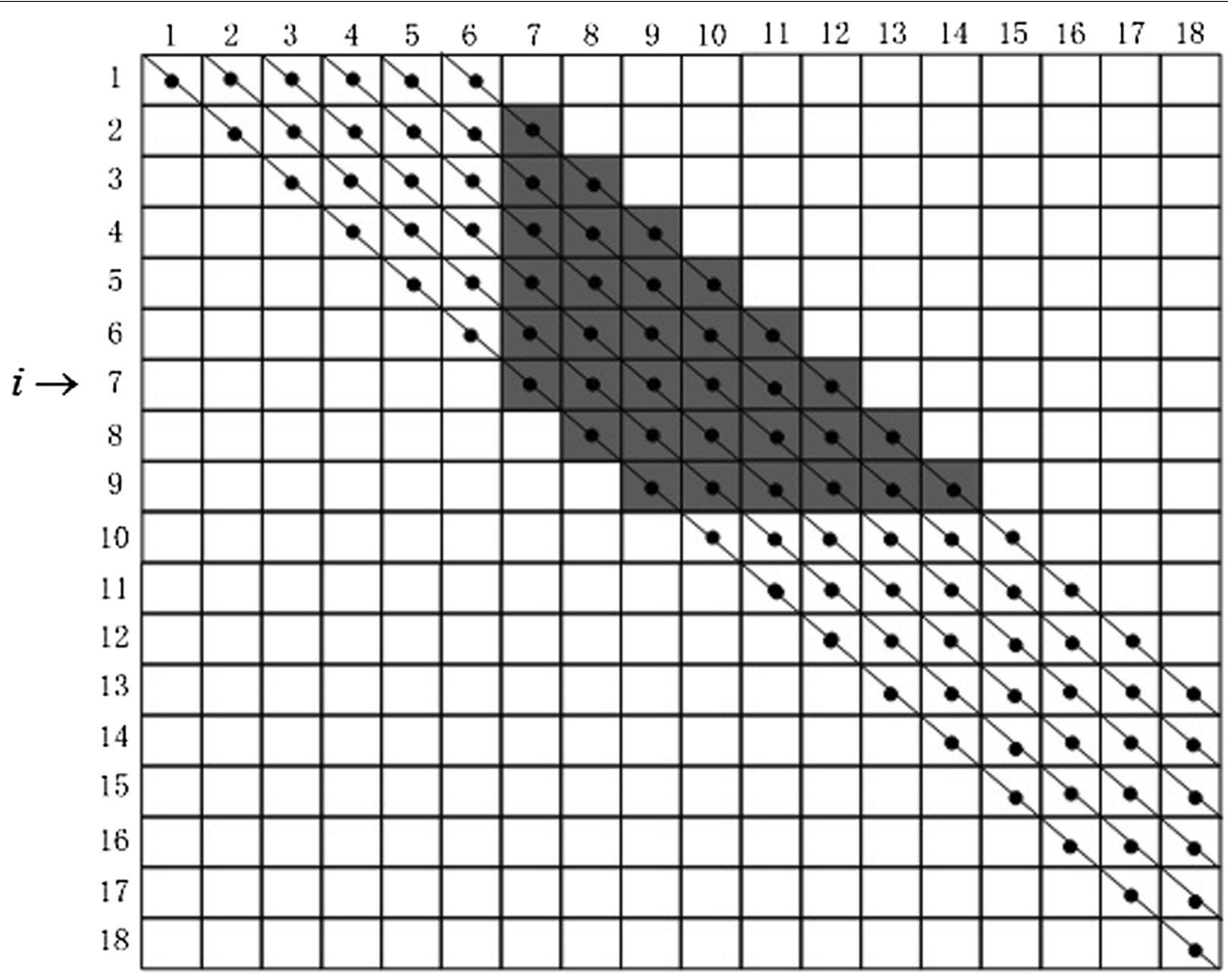

Fig. 8 Schematic diagram of the lag profile matrix 
It should be pointed out that the long pulse has poor range resolution, so many coding schemes including the barker, complementary and alternative code have been developed to meet the different requirements including the range resolution, spectra resolution and signal to noise ratio. Nowadays QJISR uses the 13-bit barker code and 16-bit alternative code, which is phase-modulated code with two phase, i.e., 0 and 180 .

Since there are several peaks and obvious side lobes in the range ambiguity function except for the zero-order lag as shown in Fig. 9, which will degrade the range resolution and signal-to-noise ratio, the barker code is only used to get the echo power profile and no autocorrelation function in more than zero lag.

The autocorrelation function of the alternative code can be represented as the formula (4). An alternative code set consists of several code sets; for example, there are $2 \mathrm{~N}$ code sets for the N-bit alternative code. Typically, each successive transmission pulse is modulated with a different code set in the sequence. In the formula (4), the different code set has different coefficients $k_{i}$ while NP is the total number of coed sets.

$<\sum_{i=0}^{\mathrm{NP}-1} k_{i} \cdot \mathrm{ACF}_{r, \tau}>=k \cdot \mathrm{ACF}_{\text {ion }}\left(t-t^{\prime}, \bar{r}\right) \int_{-\infty}^{\infty} W_{t, t^{\prime}}^{r}(S) \cdot \mathrm{ds}$

FFT is a widely used spectra estimation method, but it will result in the spectral leakage, so the proper window function is applied such as the rectangular window in QJISR.

The incoherent integration is implemented to improve the SNR and measurement performance since the measurement error is mainly related to the SNR. Evans (1969) found that when the SNR fall below unity, the percentage error increases rapidly and it is impossible to achieve an accuracy better than $10 \%$ in a reasonable time (e.g., $10 \mathrm{~min}$ ) for $\mathrm{SNR}<0.1$. When the SNR is more than 1 , it is possible to determine the scatted echo power to an accuracy of $1 \%$ in about $5 \mathrm{~min}$. The integration time is usually above 5000, which corresponding to $1 \mathrm{~min}$ with the pulse repetition period of $12 \mathrm{~ms}$.

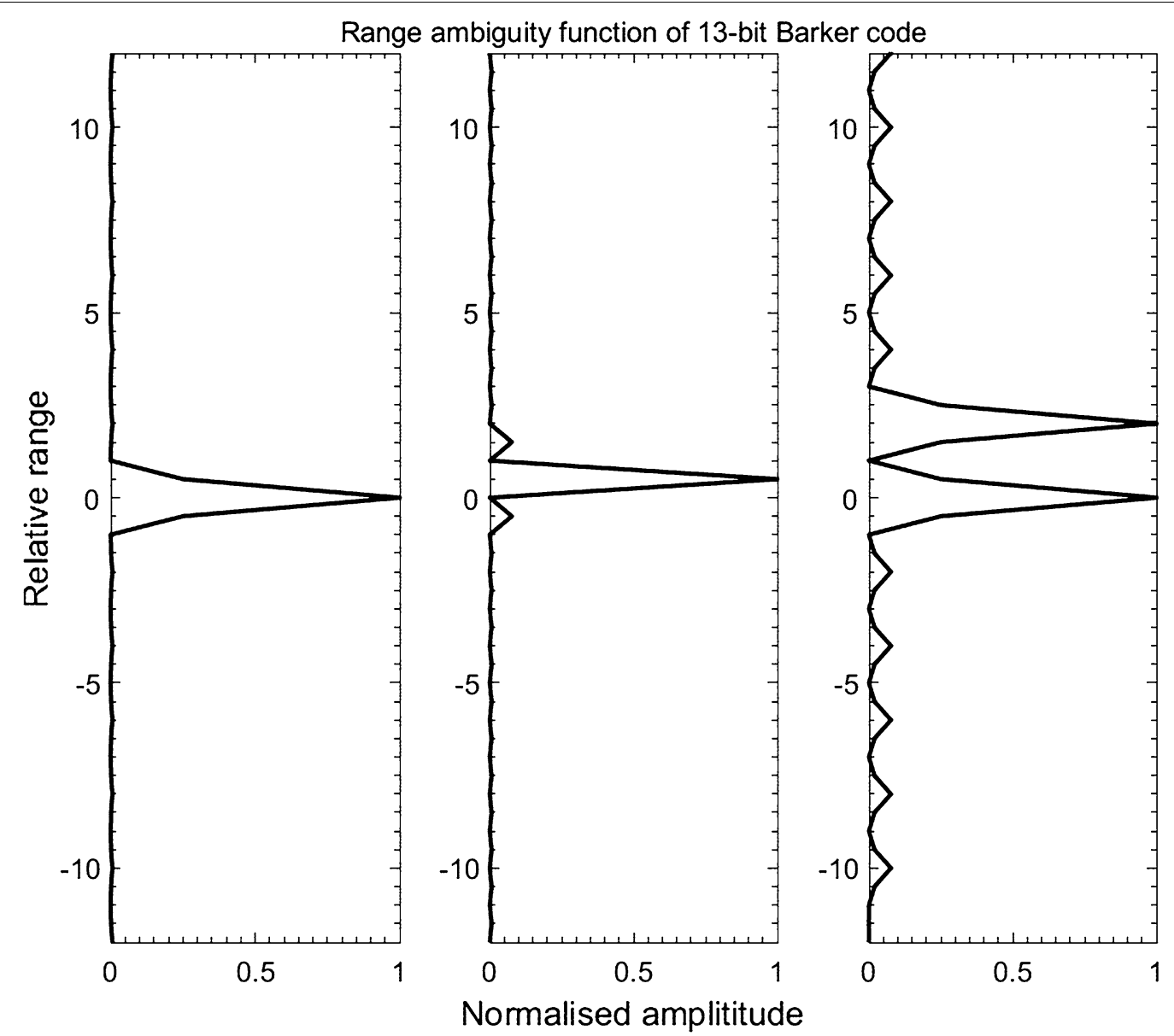

Fig. 9 Range ambiguity function of the 13-bit barker code 
The range gate is usually set between 100 and $800 \mathrm{~km}$ in altitude. The echo signals less than $100 \mathrm{~km}$ are sometimes contaminated by the clutter or meteor and the $F$ region beyond $800 \mathrm{~km}$ signals often become unusable owing to the weak signal strength.

\section{Ionospheric parameter inversion}

The above echo profile and power spectra form the basis of the subsequent data inversion and are stored in the local terminal server by a local network. The principal function of the local server consist of: (1) storing the above measured data; (2) real-time display the data in the graphics format; (3) data inversion to get the ionospheric parameters including the electron density, electron and ion temperature, plasma drift velocity.

The echo power $P_{\mathrm{r}}$ is related to the electron density $N_{\mathrm{e}}$ as the formula (5) where $C$ is the radar system constant determined by the radar power, wavelength, system noise temperature, pulse width, receiver bandwidth (Ding et al. spectra (Evans 1969) in which one is listed as formula (6). The $\omega$ is the angular frequency and $K$ is the Boltzmann constant. The $D_{\mathrm{e}}$ and $D_{\mathrm{i}}$ are the electron and ion Debye length, respectively. The $T_{e}$ and $T_{\mathrm{i}}$ are the electron and ion temperature. The $m_{\mathrm{e}}$ and $m_{i}$ are the electron and ion mass. $\overline{\left|N_{\mathrm{e}}(w)\right|^{2}}$ and $\overline{\left|N_{\mathrm{i}}(w)\right|^{2}}$ are the fluctuation spectrum of independent electrons and ions. The spectra can be seen as containing two terms: electronic component and ionic component. When the Debye length is far less than radar wavelength, the amount of power in the electronic component decreases and appears in a single line at a Doppler shift approximately equal to the plasma frequency for the medium (called plasma line).The ionic component is also called ion line. Actually, it is difficult to detect the plasma-line echo since the amount of power associated with this line is far less than that of the ion line. So the QJISR only measures the ion line at present. The ion-line spectrum inversion of QJISR is based on the ion-line spectral as formula (6).

$$
S(\omega)=\frac{\left|1+\left(\frac{\lambda}{4 \pi}\right)^{2} \cdot \sum_{i} \frac{F_{i}(\omega)}{D_{i}^{2}}\right|^{2} \cdot\left|\overline{N_{\mathrm{e}}(w)}\right|^{2}+\left(\left(\frac{\lambda}{4 \pi D_{\mathrm{e}}}\right)^{4}\right) \cdot\left|F_{\mathrm{e}}(\omega)\right|^{2} \cdot \sum_{i}\left|\overline{N_{\mathrm{i}}(w)}\right|^{2}}{\left|1+\left(\frac{\lambda}{4 \pi}\right)^{2} \cdot\left\{\frac{F_{\mathrm{e}}(\omega)}{D_{\mathrm{e}}^{2}}+\sum_{i} \frac{F_{i}(\omega)}{D_{i}^{2}}\right\}^{2}\right|}
$$

2014b). For the QJISR, the $C$ vary daily but remain nearly constant more than $10 \mathrm{~h} . R$ is the range from the radar to the scattering volume of ionospheric, $T_{\mathrm{r}}$ is the ratio of electron to the ion temperature and $\alpha$ is the square of the ratio of Debye length to the radar wavelength. The wavelength of QJISR is $60 \mathrm{~cm}$, and the Debye length of ionospheric plasma is less than centimeters, so the Debye factor is negligible. We usually normalize the measured echo profile at its peak to the F layer peak density measured simultaneously by a co-located ionosonde to derive the electron density profiles. This profile called 'Raw electron density' is accurate at the $F$ layer peak but at any other height is in error mainly by the $T_{\mathrm{r}}$, which could lead to a factor of 2 in the worst case.

$$
P_{\mathrm{r}}=\frac{C N_{\mathrm{e}}}{R^{2}\left(1+T_{\mathrm{r}}+\alpha\right)(1+\alpha)}
$$

The measured echo power actually incorporates both the ionospheric signal and background noise. So it is necessary to remove the background noise from the measured echo power. Since the ionospheric echo power above $700 \mathrm{~km}$ is usually very weak due to less electron content and farther range, the measured minimal power above $700 \mathrm{~km}$ is thought of as the noise power level for QJISR.

The formula of power spectra related to ionospheric parameters is very complex. Several researchers had studied the ionospheric incoherent scatter theory and obtained the similar expressions of incoherent scatter

$$
\begin{aligned}
& F_{\mathrm{e}}(\omega)=1-\omega \int_{0}^{\infty} \exp \left(-\frac{16 \pi^{2} K T_{\mathrm{e}}}{\lambda^{2} m_{\mathrm{e}}} \tau^{2}+\omega \tau\right) \cdot \mathrm{d} \tau \\
& F_{\mathrm{i}}(\omega)=1-\omega \int_{0}^{\infty} \exp \left(-\frac{16 \pi^{2} K T_{\mathrm{i}}}{\lambda^{2} m_{\mathrm{i}}} \tau^{2}+\omega \tau\right) \cdot \mathrm{d} \tau \\
& \overline{\left|N_{\mathrm{e}}(w)\right|^{2}}=2 N_{\mathrm{e}} \int_{0}^{\infty} \exp \left(-\frac{16 \pi^{2} K T_{\mathrm{e}}}{\lambda^{2} m_{\mathrm{e}}} \tau^{2}\right) \cdot \cos (\omega \tau) \cdot \mathrm{d} \tau
\end{aligned}
$$

It should be noted that the above method assumes that the electrons and ions independent of each other have the Maxwellian velocity distribution. But the non-Maxwellian distribution characteristics can be induced by intensive convective electric fields in the high latitude, energetic particle precipitation in the aurora as well as by artificial ionospheric heating.

Using the nonlinear fitting algorithm such as the Marquart-Levenberg method (Nygren 1996), the ionospheric parameters can be obtained from the measured power spectra. The ionospheric parameter inversion of QJISR is performed using a MATLAB software modified from the GUISDAP package (Lehtinen and Huuskonen 1996) with the help of EISCAT staff. 


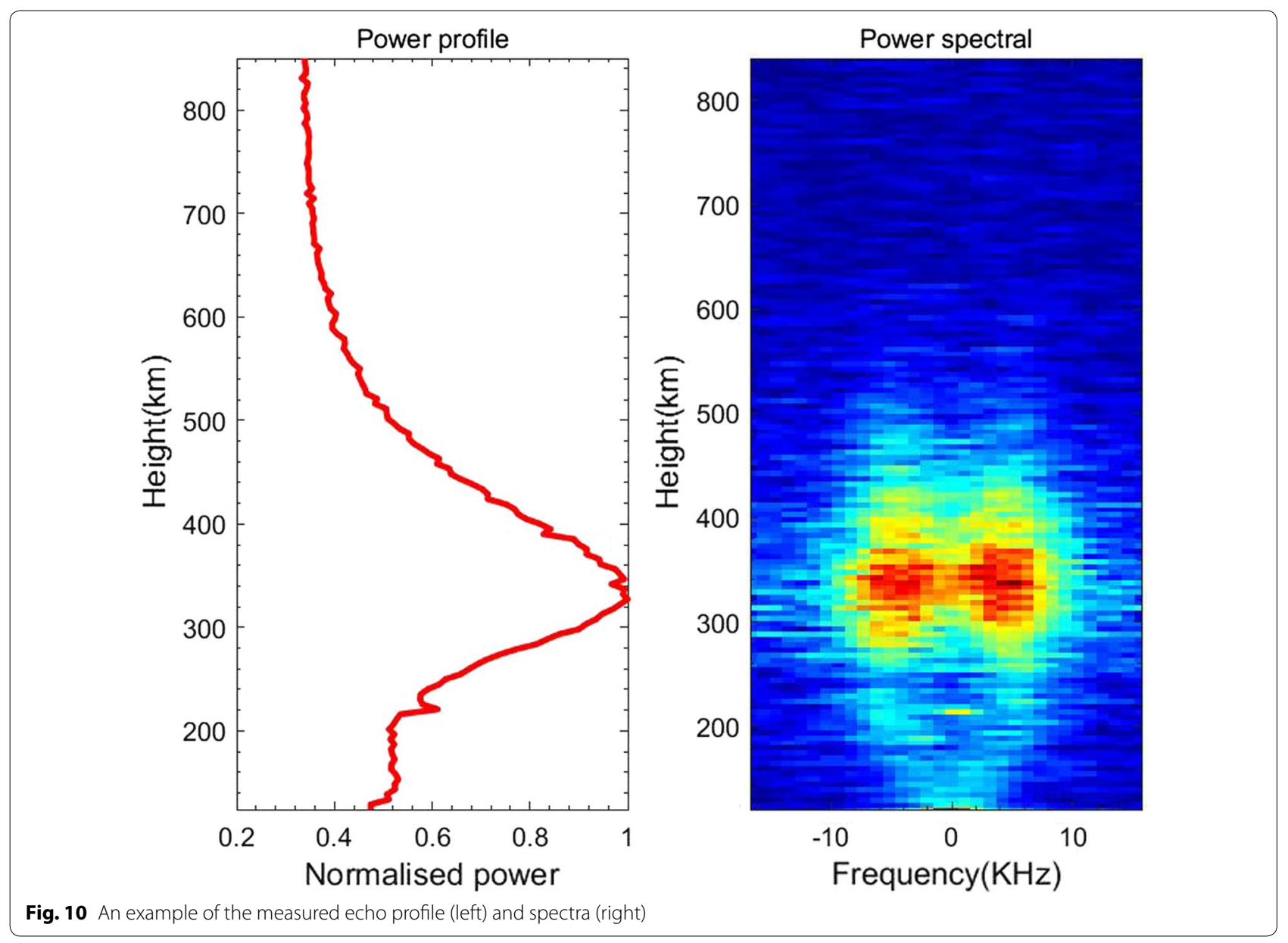

\section{Preliminary observations}

From the spring of 2014, the QJISR have run for several hundreds of hours and large amount of data is obtained. As described above, the barker code pulse is used to measure the echo profile and inverse the raw electron density. The alternating code is used to measure the power spectra and inverse the ionospheric parameters.

Figure 10 shows one measured ionospheric echo profile and one power spectra, which is normalized by the maximal echo and power spectra value. Since the echo above $550 \mathrm{~km}$ is very weak due to less electrons and farther range, its SNR is too low to be detectable and so it sets the upper height at which the reliable profiles can be obtained.

Figure 11 shows the daily variation of the raw electron density in November, 2014. In this measurement, the used pulse repetition period is $12 \mathrm{~ms}$. The pulse width is 390 us, and the baud width is 30 us, which means that the range resolution is $4.5 \mathrm{~km}$. The integration time for each measurement is 10,000 , which is equal to $120 \mathrm{~s}$. The electron density is in units of $10^{10} / \mathrm{m}^{3}$. It can be found that the electron density and height of F2 layer increase gradually with the local time and reach the apex in 13:00LT, which represents the normally daily variation of the ionosphere at Qujing site. It needed to be pointed out that the F2 peak altitude (ab. hmF2) is higher during daytime than nighttime, which is different form that at mid-latitude site. The main mechanism of the local time variation of the $\mathrm{hmF} 2$ is the electric field and neutral wind (Su et al. 1997). At the mid-latitude site such as MU radar, the effect of the pole-ward neutral wind is stronger than the effect of the eastward electric field during daytime and so the electron density is lower at noon than during the morning and at pre-sunset periods. During nighttime, equator-ward neutral wind pushes up the F-region to higher altitudes along the magnetic field line through the neutral-ion collision. The typical local time variation of the hmF2 is that the F2 peak altitude is lower during daytime and higher during nighttime at the mid-latitudes. Zhang et al. (1999) have discussed the $\mathrm{hmF} 2$ variations using the measurement by the MU radar $\left(34.86^{\circ} \mathrm{N}, 136.18^{\circ} \mathrm{E}\right)$. It is found that $\mathrm{hmF} 2$ is generally higher during the periods of the solar maximum than the solar minimum and higher in summer than in winter. 


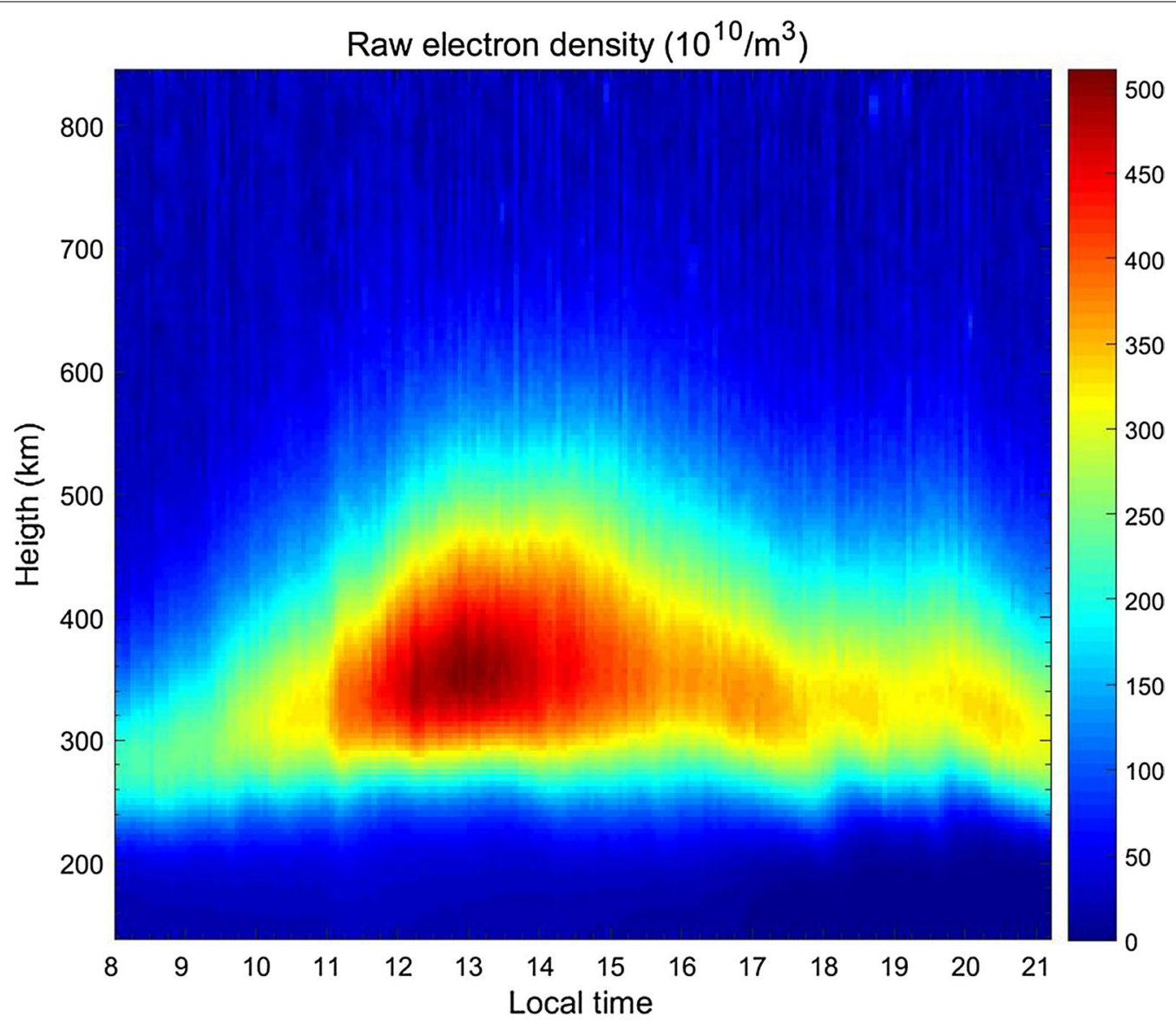

Fig. 11 Daily variation of raw electron density in November 2014

During the solar maximum, hmF2 drops markedly in the morning and afternoon, while for the solar minimum, the hmF2 minimum occurs in the morning during summer and usually in the afternoon during winter. The neutral wind contributes strongly to the diurnal variation of hmF2 in winter by lowering the ionization layer by day, particularly for the solar maximum. Oliver et al. (2008) think that the diurnal variation of hmF2 is driven most importantly by winds, secondarily by thermal expansion. At geomagnetic equator, the electric field is the main source to control the ionospheric F2 dynamical features. The QJISR site is located between the equator and midlatitude and also around the northern crest of the equatorial ionization anomaly. The relative importance of the electric field and neutral wind on the ionospheric $F$ region seem different from the typical mid-latitude site.

Figure 12 shows the local-time variation of the raw electron density in April 2017. It needs to be noted that there occurred the enhancement of electron density after sunset during the local time 17:00-18:00. Simultaneously, the height of F2 layer starting at about $300 \mathrm{~km}$ gradually decreased from 16:30 and reached the minimum $260 \mathrm{~km}$ at 19:00LT. The change of hmF2, which is about $-40 \mathrm{~km}$, represents the downward ion drift of F2 layer that may be caused by the westward electric field. It is well known that the electron density will decrease with the increasing of the neutral atmosphere density and recombination coefficient with the lowering of F2 layer. In this sunset enhancement event, the electron density increases sharply with the lowering of F2 layer, which mean that more process including the electric field, neutral wind, plasma diffusion and the related EIA processes cause this event, of which the details will be investigated in the future with the support of more data.

Figure 13 shows the daily variation of the ionospheric electron density, electron temperature, ion temperature, and plasma drift velocity. Due to the low SNR for the spectra above $550 \mathrm{~km}$, the error of ionospheric parameters is significant, and so the ionospheric parameters above $550 \mathrm{~km}$ are not shown. In this measurement, the used pulse repetition period is $12 \mathrm{~ms}$. The pulse width is 480us, and the 16-bit sub-pulse width is 30us. During the inversion, the spectral is moving averaged in both the range and time to get better accuracy. The resulting 


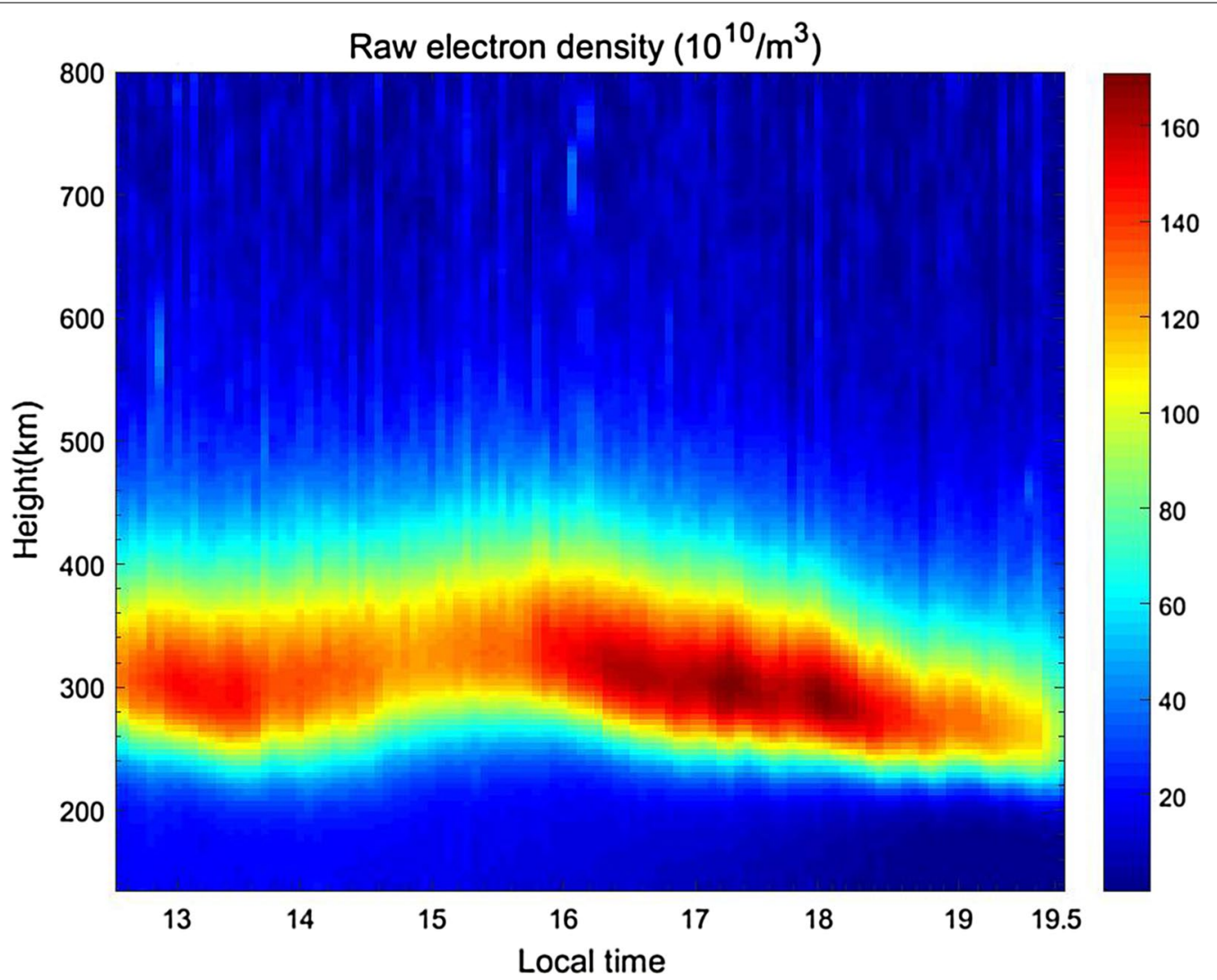

Fig. 12 Daily variation of raw electron density in April 2017

time and height interval for each measurement in Fig. 13 are about $5 \mathrm{~min}$ and more than $15 \mathrm{~km}$ dependent of the height. To improve the inversing accuracy, we take the different height interval at different height, for example the height interval is $10-20 \mathrm{~km}$ below $300 \mathrm{~km}$, but it is $25-40 \mathrm{~km}$ above $400 \mathrm{~km}$. This method is feasible and widely used by EISCAT since the ionospheric-scale height varies with the height.

The electron temperature has a maximum at around $220 \mathrm{~km}$ and decreases to the minimal value at about $310 \mathrm{~km}$, and then it will increase again. This is because the electron heating rate in the $\mathrm{F}$ region is proportional to the plasma density, while the electron cooling rate due to Coulomb collisions with the ions is proportional to square of the plasma density. The maximal electron temperature of F2 layer in daytime is above $2000 \mathrm{~K}$ and will decrease sharply after sunset. It seems that there exists an overshoot of the electron temperature at about the F2 peak altitude in the morning $(\sim 9 \mathrm{LT})$ and sunset $(\sim 18$ LT). The overshoot of the electron temperature occurs when the plasma density is low but the solar heating exists because cooling rate of the electron is proportional to the plasma density in the ionosphere. At sunrise, photoelectron production begins in the ionosphere through the ionization of neutral particles. As the photoelectrons share their high energy with the ambient electrons, the electron temperature increases; the increase is rapid in the early morning due to the low electron density. As more and more electrons are produced with the progress of the morning, the energy share for each electron decreases. Thus, the electron temperature, after reaching a maximum, decreases and attains a steady value by the end of the morning (Otsukaet al. 1998; Oyama et al. 1996; Watanabe et al. 1995). At sunset, the overshoot of electron temperature is related to the electron density and its variation during the afternoon-evening period. During this period, the electron density generally decreases with time. The decrease will become rapid if the neutral wind is pole-ward. The wind forces the ionosphere to descend to low altitudes full of heavy chemical loss, which causes the rapid decrease of electron density before sunset. The temperature of the electron gas should increase before sunset because of photoelectron heating in the same way as the morning increase in electron temperature (Otsuka et al. 1998; Watanabe et al. 1995). Lei et al. (2007) have found that the diurnal variation of Te is characterized by morning and evening peaks at Arecibo $\left(18.3^{\circ} \mathrm{N}, 66.7^{\circ} \mathrm{W}\right)$ and by a morning peak at Millstone Hill $\left(42.6^{\circ} \mathrm{N}, 71.5^{\circ} \mathrm{W}\right)$. 


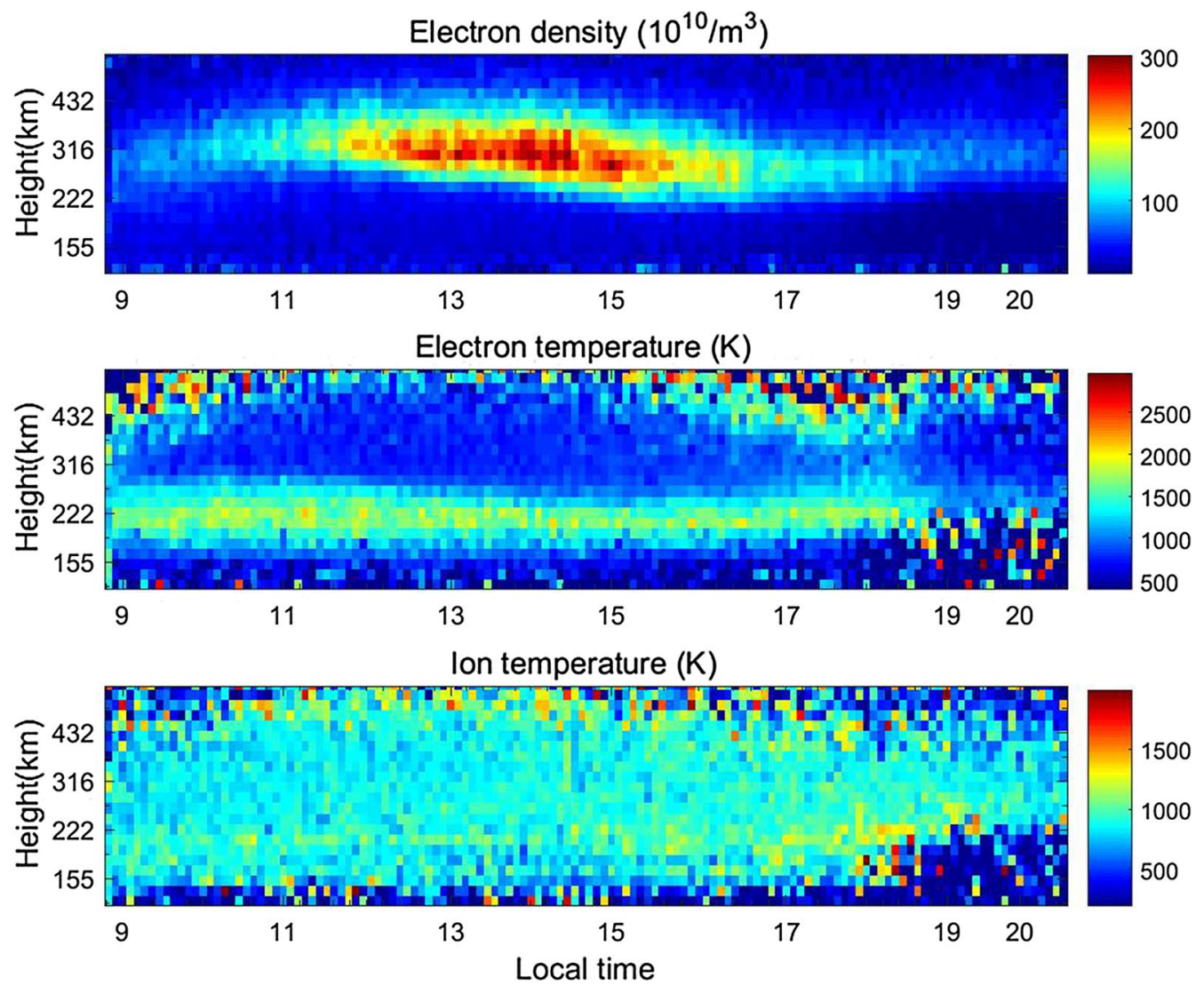

Fig. 13 Temporal variation of ionospheric parameters on May, 2017 (from the top to end: electron density, electron temperature and ion temperature)

The occurrence and strength of the peaks at Arecibo are significantly different from those at Millstone Hill. The simulations show that the daytime bulge of Te tends to occur at low latitudes and high solar activity and the significant morning peak at low solar activity over Arecibo is associated with the equatorial anomaly. The daily variation of Te in Fig. 13 seems a bit similar to that at Arecibo but needs further investigations.

The ion temperature increases below $200 \mathrm{~km}$ because the ions are essentially in thermal equilibrium with the neutrals due to the large ion-neutral collision frequency. And then the ion temperature slowly increases above $200 \mathrm{~km}$ and finally approaches the electron temperature above $600 \mathrm{~km}$ where the electron-ion collisions dominate.

Since the velocity data measured used the above method is not quite reliable or not mature, we disregard it. In the future, we will try to estimate the mean Doppler line of sight (due to only one beam, which is fixed) velocity by fitting the phase variation of observed ACF with time, which is similar to the method as suggested in Sato et al. (1989).

\section{Summary}

Although QJISR may be considered as a Generation One of incoherent scatter radar system, while the current state of the art is at Generation Three, the QJISR is thought of be important for its scientific merits due to be as the first one constructed in China and its unique location of the radar site. In this paper we have described its principal technical features. Its main technical parameters include the peak power $2 \mathrm{MW}$, the parabolic dish with the aperture $29 \mathrm{~m}$, and frequency $500 \mathrm{MHz}$.

The QJISR may act as an important tool for the ionosphere measurements over the southwestern China. Some possible scientific cases include: (1) structure and evolution of the EIA; (2) coupling between the mid-low atmosphere and ionosphere; (3) ionosphere modeling. In addition, it is possible to observe the ionospheric irregularity with the beam perpendicular to the geomagnetic filed. With the beam directing to south, it is also possible to measure the coherent echo from the plasma bubble originated at geomagnetic equator.

The data processing and inversion of ISR measurements are highly complicated, so the inversion method 
and its accuracy analysis of QJISR measurement need great effort in the future.

\section{Abbreviations}

EISCAT: European incoherent scatter radar associate; ISR: incoherent scatter radar; ACF: autocorrelation function; GUISDAP: grand unified incoherent scatter design and analysis package.

\section{Authors' contributions}

DZH made substantial contributions to conception, data analysis, data interpretation, and manuscript drafting. WJ participated in the conception, coordination, and manuscript revision. XZW assisted in the drafting and revising this manuscript. XB participated in the data analysis. DLD participated in the measurements and data collecting. All authors read and approved the final manuscript.

\section{Acknowledgements}

The authors are grateful to Dr. Ingemar Haggstrom (EISCAT) for his great help in the data analysis software development and usage, and thank the Meridian Project of China for their funding in the radar operation and data collection. Also many thanks for the two anonymous reviewers and the editor for their important suggestion in the manuscript revision.

\section{Competing interests}

The authors declare that they have no competing interests.

\section{Ethics approval and consent to participate}

Not applicable.

\section{Publisher's Note}

Springer Nature remains neutral with regard to jurisdictional claims in published maps and institutional affiliations.

Received: 28 September 2017 Accepted: 15 May 2018

Published online: 25 May 2018

\section{References}

Bowles KL (1958) Observation of vertical incidence scatter from the ionosphere. Phys Rev Lett 1:454-455

Ding ZH, Dai LD, Dong MY, Xu ZW, Wu J (2014a) Progress of the incoherent scattering radar: from the traditional radar to the latest EISCAT 3D. Progress in Geophysics 29(5):2376-2381 (in Chinese)

Ding ZH, Yu L, Dai L, Xu ZW, Wu J (2014b) The preliminary measurement and analysis of the power profiles by the Qujing incoherent scatter radar. Chin J Geophys 57:3564-3569

Evans JV (1969) Theory and practice of ionosphere study by Thomson scatter radar. Proc. Of IEEE 57(4):494-530

Evans JV (1970) Millstone Hill Thomson scatter results for 1965. Planetary Space Sci 18(8):1225-1253

Farley DT (1991) Early incoherent scatter observations at Jicamarca. J Atmos Terr Phys 53:665-675

Gordon WE (1958) Incoherent scatter of radio waves by free electrons with applications to space exploration by Radar. Proc IRE 46(11):1824-1829

Huuskonen A, Kangas J, Turunen T (1987) EISCAT as a radar for auroral research a case study. Geophysica 23:35-46

loannidis G, Farley DT (1972) Incoherent scatter observations at Arecibo using compressed pulses. Radio Sci 7(7):763-766
Kelly JD, Heinselman CJ, Vickrey JF (1995) The Sondrestrom radar and accompanying ground-based instrumentation. Space Sci Rev 71(1-4):797-813

Lehtinen MS, Haggstrom I (1987) A new modulation principle for incoherent scatter measurements. Radio Sci 22(4):625-634

Lehtinen MS, Huuskonen A (1996) General incoherent scatter analysis and GUISDAP. J Atmos Terr Phys 58:435-452

Lei JH, Roble RG, Wang W, Emery BA, Zhang SR (2007) Electron temperature climatology at Millstone Hill and Arecibo. J Geophys Res 112:A02302. https://doi.org/10.1029/2006JA012041

Markkanen M, Vierinen J, Markkanen J (2008) Polyphase alternaing codes. Ann Geophys 26:2237-2243

Nygren T (1996) Introduction to incoherent scatter measurements. INVERS Publication, Finland. ISBN 951-97489-0-3

Oliver WL, Kawamura S, Fukao S (2008) The causes of mid-latitude F layer behavior. J Geophys Res 113:A08310. https://doi.org/10.1029/2007」 A012590

Otsuka Y, Kawamura S, Balan N, Fukao S, Bailey GJ (1998) Plasma temperature variations in the ionosphere over the middle and upper atmosphere radar. J Geophys Res 103(A9):20705-20713

Oyama KI, Balan N, Watanabe S, Takahashi T, Isoda F, Bailey GJ, Oya H (1996) Morning overshoot of Te enhanced by downward plasma drift in the equatorial topside ionosphere. J Geomagn Geoelectr 48:959-966

Sato T, Ito A, Oliver WL, Fukao S, Tsuda T, Kato S, Kimura I (1989) lonospheric Inchoherent Scatter Measurements with the MU Radar: techniques and capability. Radio Sci 24:85-98

Su YZ, Fukao S, Bailey GJ (1997) Modeling studies of the middle and upper atmosphere radar observations of the ionospheric F layer. J Geophys Res 102(A1):319-327

Sulzer MP (1993) A new type of alternating code for incoherent scatter measurements. Radio Sci 28(6):995-1001

Susumu K, Ogawa T, Tsuda T, Sato T, Kimura I, Fukao S (1984) The middle and upper atmosphere radar: first results using a partial system. Radio Sci 19(6):1475-1484

Wang C (2010) New chains of space weather monitoring stations in China. Space Weather. https://doi.org/10.1029/2010SW000603

Wannberg G, Wolf I, Vanhainen LG, Koskenniemi K, Rottger J, Postila M, Maekkanen J, Jacobsen R, Stenberg A, larsen R, Heck S, Huuskonen A (1997) The EISCAT Svalbard radar: a case study in modern incoherent scatter radar system design. Radio Sci 32(6):2283-2307

Watanabe S, Oyama Kl, Abdu M (1995) A computer simulation of electron and ion densities and temperature in the equatorial $\mathrm{F}$ region and comparison with Hinotori results. J Geophys Res 100(14):581

Ya EL, Zhivolup TG (2013) History of the development of IS radars and founding of the Institute of lonosphere in Ukraine. Hist Geo Space Sci 4:7-17

Zhang SR, Fukao S, Oliver WL, Otsuka Y (1999) The height of the maximum ionospheric electron density over the MU radar. J Atmos Solar Terr Phys 61(18):1367-1383

Zhao B, Wang M, Wang Y, Ren ZP, Yue XA, Xiong B (2013) East-west differences in F-region electron density at mid-latitude: evidence from the Far East region. J Geophys Res 118(1):542-553

\section{Submit your manuscript to a SpringerOpen ${ }^{\circ}$ journal and benefit from:}

- Convenient online submission

- Rigorous peer review

- Open access: articles freely available online

- High visibility within the field

- Retaining the copyright to your article

Submit your next manuscript at springeropen.com 